\title{
A Heat Transfer Model for Accurate Wellbore Temperature Prediction during Drilling
}

\author{
Ming Jiang* \\ CNPC Bohai Drilling Company
}

*Corresponding Author: Ming Jiang, CNPC Bohai Drilling Company

\begin{abstract}
Accurate prediction of wellbore temperature is essential to avoid potential drilling fluid/drillstring overheating and wellbore stability problems. A new model is proposed to predict the dynamic wellbore temperature during drilling. Besides the effect of fluid flow on the thermal transfer, the model also incorporates effects caused by the forces created from the contacts between drillpipe and casing/formation. The new model is utilized in a practical well to study the dynamic temperature changes. The model can be used to accurate prediction temperature profiles in the wellbore at the design stage or mud and downhole tools selection during drilling operation.
\end{abstract}

Keywords: heat transfer, wellbore temperature, drill string friction, numerical method

\section{INTRODUCTION}

Prediction of fluid temperature in the wellbore is required to ascertain the fluid density and viscosity, and in turn to calculate the pressure-drop or the maximum allowable pumping rate for a number of operations. Borehole temperature is also a significant parameter in determining wellbore stability. Overheating causes substantial change to rock stress, which has a subsequent effect on wellbore stability, which may lead to loss of drilling fluid circulation, stuck drillpipe (which requires costly operation, e.g., side tracking) and other drilling problems. A number of wellbore heat transfer studies have been conducted, and they can be classified into two categories: analytical methods, and numerical methods.

Analytical methods require less time and energy, but are not as accurate as numerical methods, because analytical methods require additional assumptions and simplifications to reduce the calculations. Therefore, analytical methods may be restricted to simpler geometries. For most engineering problems, analytical methods are usually the first approaches to reach for a solution.

An early analytical model ${ }^{14}$ to predict circulating fluid temperature assumes steady-state linear heat transfer between the annular fluid and the formation, which was not widely applied due to the steadystate assumption. Later, Hasan et al. ${ }^{6}$ (1996) improved the previous model by taking into account the variable fluid inlet temperature and variable heat flux. Karstad and Aadnøy ${ }^{11}$ (1997) investigated the temperature effects during drilling and expanded previous models to be able to handle both an increasing well depth and a variable mud-in temperature, but, it is only valid for short time frames (i.e., the drilling phase). Karstad ${ }^{10}$ (2006) provided a more comprehensive and improved model to predict circulating fluid temperature (backward and forward circulation) and bottomhole pressure during drilling operation. This model also considers the effects of riser, hydraulics and rotation on offshore wellbore heat transfer.

Compared to analytical method, numerical methods are more time-consuming and require extensive data input, but they provide relatively accurate predictions. One of the first numerical models to calculate drilling fluid temperature profile as a function of time was developed by Raymond ${ }^{17}(1969)$ using a finite difference method. Keller et al. ${ }^{12}$ (1973) improved the previously available models by considering multiple casing strings in the completion layout, which provided more flexibility. Wooley $^{23}$ (1980) then developed the model to predict wellbore temperatures in different operating conditions. This method does not decouple soil and well calculations like previous models, it simultaneously computes temperatures in the two regions, which can improve speed and accuracy. 
Chen and Novotny ${ }^{3}$ (2003) developed a numerical simulator to predict wellbore temperature profiles in wells with multiple temperature gradients and wellbore deviations.

Most of the previous studies ignores mechanical friction, which can be a main heat source contributing to the overheating of drilling fluids. In deviated and horizontal wells, the drill string is normally in contact with the borehole wall, especially in high dogleg sections. Actual applications indicate that a tremendous amount of heat can be generated from the drill string. This study propose a heat transfer model for wellbore temperature prediction by considering the mechanical frictions. An analysis of frictional heat sources for different operations is also presented, which would be useful to minimize costly problems caused by overheating during drilling.

\section{NUMERICAL MOdEL FOR WELlbore HeAT TranSFER}

In this model, all heat transfer processes are assumed to be transient. The frictional heat source is included locally at each segment. The assumptions of the model include:

- Incompressible fluid with constant properties;

- No radiation heat transfer;

- Formation properties are independent of temperature;

- Radial temperature gradient of drilling fluid is negligible;

- The pore fluid is assumed to have Newtonian properties;

- The flow in the formation is in the radial direction only;

- Heat generated from viscous flow is neglected because it is insignificant compared to mechanical friction.

A control volume method can be used to analyze the heat exchanges between the fluids in the annulus, inside the drillpipe, and the formation, as shown in Figure 1:

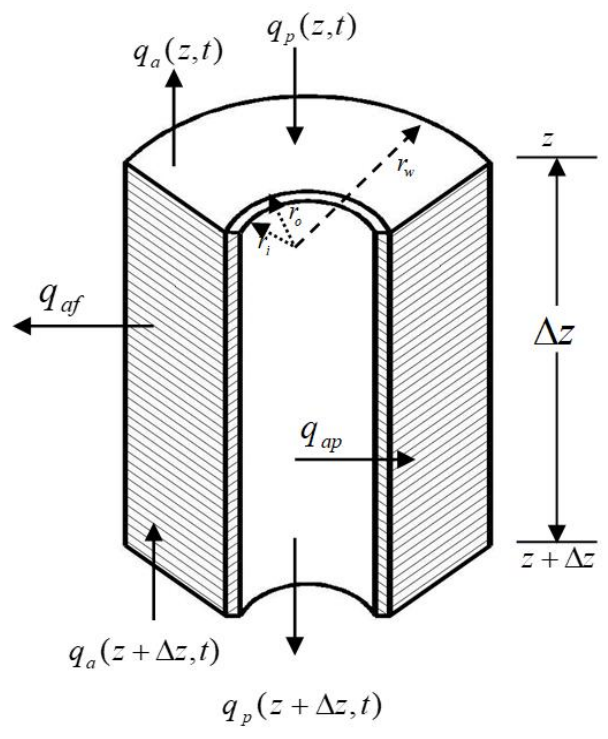

Figure1. Control volume for heat rates

The heat exiting the annulus can be formulated as:

$q_{a}(z, t)=m c_{p, m} T_{a}(z, t)$

The heat entering the annulus is:

$q_{a}(z+\Delta z, t)=m c_{p, m} T_{a}(z+\Delta z, t)$

The heat transfer from annulus to formation is:

$q_{a f}=2 \pi r_{f} h_{a}\left(T_{a}-T_{f}\right) \Delta z$

The heat transfer from drill pipe to annulus involves convection heat transfer by tubular drilling fluid 
and conduction heat transfer across the drillpipe wall. The electrical analogy is used to simulate heat flow from drillpipe to annulus, which can be illustrated by Figure 2 .

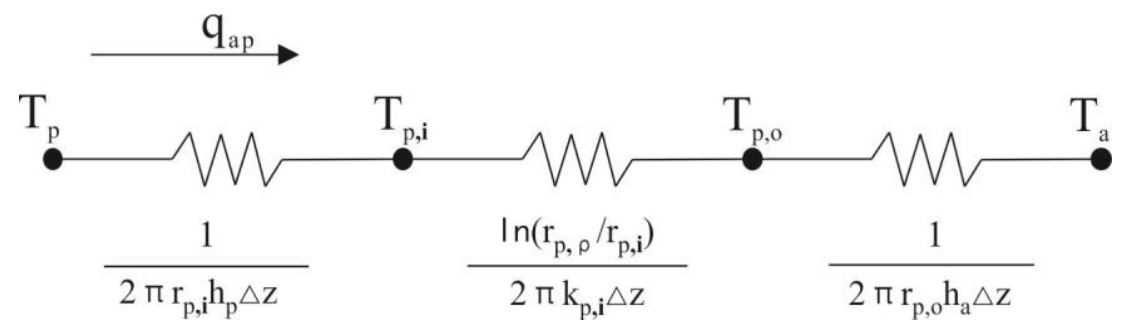

The following relationship can be obtained:

$$
q_{a p}=\frac{T_{p}-T_{a}}{\frac{1}{2 \pi r_{p, i} h_{p} \Delta z}+\frac{\ln \left(r_{p, o} / r_{p, i}\right)}{2 \pi k_{p} \Delta z}+\frac{1}{2 \pi r_{p, o} h_{a} \Delta z}}=2 \pi r_{p, i} U_{a p}\left(T_{p}-T_{a}\right) \Delta z
$$

Where

$$
U_{a p}=\frac{1}{\frac{r_{p, o}}{r_{p, i} h_{p}}+\frac{r_{p, o} \ln \left(r_{p, o} / r_{p, i}\right)}{k_{p}}+\frac{1}{h_{a}}}
$$

The change in internal energy with time is:

$q_{E a}=\rho_{m} c_{p, m} A_{a} \frac{\partial T_{a}}{\partial t} \Delta z$

The heat balance in the annulus can be presented as:

$$
\left\{\begin{array}{l}
\text { Heat entering } \\
\text { the annulus }
\end{array}\right\}+\left\{\begin{array}{l}
\text { Heat transfer } \\
\text { from drillpipe } \\
\text { to annulus }
\end{array}\right\}=\left\{\begin{array}{l}
\text { Heat exiting } \\
\text { the annulus }
\end{array}\right\}+\left\{\begin{array}{l}
\text { Heat transfer } \\
\text { from annulus } \\
\text { to formation }
\end{array}\right\}+\left\{\begin{array}{l}
\text { Change in } \\
\text { internal } \\
\text { energy }
\end{array}\right\}
$$

Which is equivalent to the following equation:

$q_{a}(z+\Delta z, t)+q_{a p}+q_{s}=q_{a}(z, t)+q_{a f}+q_{E a}$

Upon rearrangement, we can obtains:

$A_{1} \frac{\partial T_{a}}{\partial t}=A_{2} \frac{\partial T_{a}}{\partial z}-\left(T_{a}-T_{f}\right)+A_{3}\left(T_{p}-T_{a}\right)+A_{4}$

Where

$$
\left\{\begin{array}{l}
A_{1}=\frac{\rho_{m} c_{p, m} A_{a}}{2 \pi r_{w} h_{a}}, \quad A_{2}=\frac{m c_{p, m}}{2 \pi r_{w} h_{a}}, \\
A_{3}=\frac{r_{p, o} U_{a p}}{r_{w} h_{a}}, \quad A_{4}=\frac{q_{s}}{2 \pi r_{w} h_{a} \Delta z} .
\end{array}\right.
$$

Similarly, the heat balance for the drillpipe can be formulated as:

$$
\begin{aligned}
& q_{p}(z, t)=q_{p}(z+\Delta z, t)+q_{a p}+q_{E p} \\
& B_{1} \frac{\partial T_{p}}{\partial t}=B_{2} \frac{\partial T_{p}}{\partial z}+\left(T_{a}-T_{p}\right)
\end{aligned}
$$

Where

$$
B_{1}=\frac{\rho_{m} c_{p, m} A_{p, i}}{2 \pi r_{p, i} U_{a p}} \text {, and } B_{2}=-\frac{m c_{p, m}}{2 \pi r_{p, i} U_{a p}}
$$

To solve equations (8) and (11), initial and boundaries conditions are required. 
The initial condition for the annulus and inside pipe temperature profiles are that it is the same as the geothermal temperature, which can be represented by:

$T_{p}(z, 0)=T_{a}(z, 0)=T_{s f}+G_{t} T V D_{z}$

(13) The boundary conditions are:

- Controlled drilling fluid temperature at the inlet:

$T_{p}(0, t)=T_{\text {inlet }}$

- No heat transfer between drillpipe and annulus fluid at the bottomhole:

$T_{p}(L, t)=T_{a}(L, t)$

The temperature profile of the formation can be obtained through the diffusivity equation with the inclusion of convection heat transfer of formation pore fluid, as illustrated in Eq-16:

$\frac{\partial T_{f}}{\partial t}+\left(\frac{\rho_{f l} c_{p, f l}}{\rho_{f} c_{p, f}}\right) v_{r} \frac{\partial T_{f}}{\partial r}=\alpha_{f}\left[\left(\frac{\partial^{2} T_{f}}{\partial r^{2}}+\frac{1}{r} \frac{\partial T_{f}}{\partial r}\right)+\frac{\partial^{2} T_{f}}{\partial z^{2}}\right]$

where $\alpha_{f}=\frac{k_{f}}{\rho_{f} c_{p, f}}$ is formation diffusivity, $k_{f}$ is formation thermal conductivity, $\rho_{f}$ is the density of formation rock, $c_{p, f}$ is the specific heat capacity of formation rock, $\rho_{f l}$ is the density of pore fluid, and $c_{p, f l}$ is the specific heat capacity of pore fluid.

Using Darcy's law, the pore fluid velocity can be expressed as:

$v_{r}=-\frac{\kappa_{f}}{\mu_{f l} \phi} \frac{\partial P}{\partial r}$

where $\mathrm{P}$ is pore pressure, $\kappa_{f}$ is the permeability of formation rock, and $\mu_{f l}$ is the viscosity of pore fluid.

Substituting Equation (17) into Equation (16) and rearranging yields:

$\frac{\partial T_{f}}{\partial t}=\alpha_{f}\left[\left(\frac{\partial^{2} T_{f}}{\partial r^{2}}+\frac{1}{r} \frac{\partial T_{f}}{\partial r}\right)+\frac{\partial^{2} T_{f}}{\partial z^{2}}\right]+C \frac{\partial P}{\partial r} \frac{\partial T_{f}}{\partial r}$

where

$C=\frac{\rho_{f l} c_{p, f l}}{\rho_{f} c_{p, f}} \frac{\kappa_{f}}{\mu_{f l} \phi}$

The following initial and boundary conditions are needed to solve equation (18) to obtain the transient formation temperature profile:

The initial condition is that the initial formation temperature is the same as the geothermal temperature:

$T_{f}(r, z, 0)=T_{s f}+G_{t} T V D_{z}$

The boundary conditions are:

- Formation temperature is the same as geothermal temperature away from the wellbore:

$T_{f}(r \rightarrow \infty, z, t)=T_{s}+G_{t} z$

Surface temperature and formation temperature at the bottomhole are constant.

\section{Mechanical Friction Heat Sources}

Friction from drillbit: A considerable amount of work has been done on this subject by various authors with different bit types. In this study, a general formula is used to estimate the heat generated at drillbit (originating from the work of Warren $^{5}$ ): 


$$
\dot{q}_{b i t}=\frac{1}{J}(1-\beta)\left(W O B^{*} R O P+2 \pi N M_{b i t}\right)
$$

where $\mathrm{J}$ is Joule's constant, $\beta$ is bit efficiency (the portion of work done by the drillbit that is used to break the rock), $W O B$ is weight on bit, $R O P$ is the rate of penetration, $N$ is rotary speed (RPM), and $M_{b i t}$ is torque at bit.

Friction from drag force between drillpipe and formation/casing: In rotary drilling, heat generated from the drag force is one of the primary components. However, the effect of this factor on wellbore temperature profiles has not been addressed in recent studies. Therefore, in order to evaluate the value and significance of drag force, a 3D drag and torque model was investigated.

Details of the drag and torque model can be found from the work of Mitchell and Miska ${ }^{26}$. The unit contact force resulting from the drag and torque model, which subsequently relates to the drag force, is then utilized to estimate the heat generated by this mechanical drag:

- In rotating mode (rotary drilling):

$\dot{q}_{\text {drag }}=\frac{1}{J} \int_{s_{1}}^{s_{2}} \mu_{f} w_{c} r_{p} 2 \pi N d s$

- In sliding mode (downhole motor drilling):

$\dot{q}_{\text {drag }}=\frac{1}{J} \int_{s_{1}}^{s_{2}} \mu_{f} w_{c} R O P d s$

where $\mathrm{J}$ is Joule's constant, $\mu_{f}$ is friction coefficient, $w_{c}$ is unit contact force, $r_{p}$ is the outer radius of drillpipe, $N$ is rotary speed (RPM), $R O P$ is the rate of penetration, and $s$ is length.

\subsection{Case Study and Verifications}

A case study is conducted by using the model discussed above to illustrate the practical application of the model. The well trajectory for the case study is shown in the following figure (Figure 2).

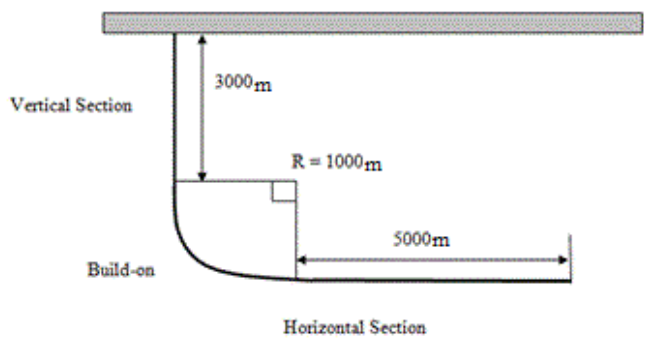

Figure2. Well trajectory

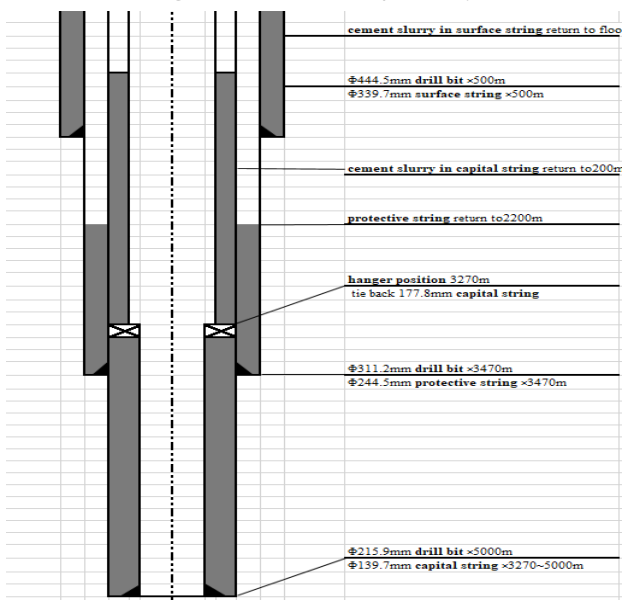

Figure3. Wellbore structure 
The wellbore structure is shown in Figure 3, and the drill string configurations are shown in Table 1. The drill string is mostly composed by $127 \mathrm{~mm}$ drill pipe and 10 drill collars with $165 \mathrm{~mm}$.

The thermal parameters of the formation, fluid, and drill string are shown in Table 2. The formation temperature gradient is $2.3{ }^{\circ} \mathrm{C} / 100 \mathrm{~m}$.

Table2. Thermal parameters for the simulation

\begin{tabular}{|c|c|c|c|}
\hline Item & Specific heat capacity $(\mathrm{J} / \mathrm{kg})$ & Thermal conductivity $(\mathrm{W} /(\mathrm{m} \mathrm{K}))$ & Density $\left(\mathrm{g} / \mathrm{cm}^{3}\right)$ \\
\hline Formation & 837 & 1.82 & 2.6 \\
\hline Drilling fluid & 1674 & 1.73 & 1.3 \\
\hline Pore fluid & 3977 & 1.73 & 0.85 \\
\hline
\end{tabular}

The simulated temperature profiles in different regions in the wellbore are shown in Figure 4. As shown in the figure, the predicted annulus mud temperature is compared to downhole measurements. It can be seen that the model prediction results is consistent with the actual measurements, which proves the accuracy of the model. Compared to the virgin formation temperature, both the mud temperature inside the drill pipe and in the annulus are higher in at the surface and lower below certain depths. And the annulus temperature is general higher than the temperature inside the drill pipe, except at the bottom of the wellbore. This represents that the mud is cooling the wellbore at downhole, and got heated by the formation before flowing back to the surface, which is also consistent with the practical process.

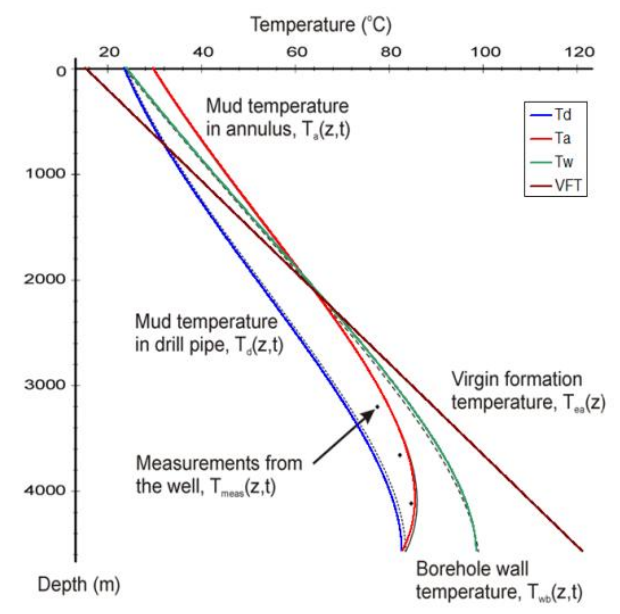

Figure4. Temperature profiles at different region of the wellbore

The temperature distributions of drilling fluid in different operating conditions are shown in Figure 5. It can be seen that bottom hole temperature reaches a very high value in rotary drilling mode. However, the majority of additional heat added is due to the drill bit during the sliding mode; the amount of frictional heat generated from drag force is negligible (3\%) compared to rotary drilling (75\%), as shown in Figure 6. This is because the rotary speed is much higher than the rate of penetration, and thus drag force in rotating mode is more significant than in sliding mode.

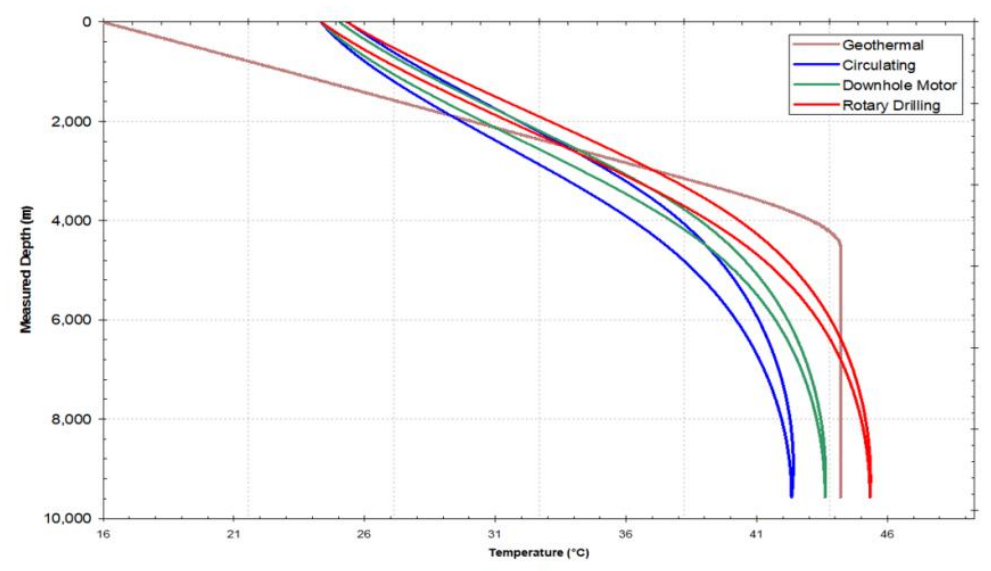

Figure5. Temperature profile at different drilling mode 


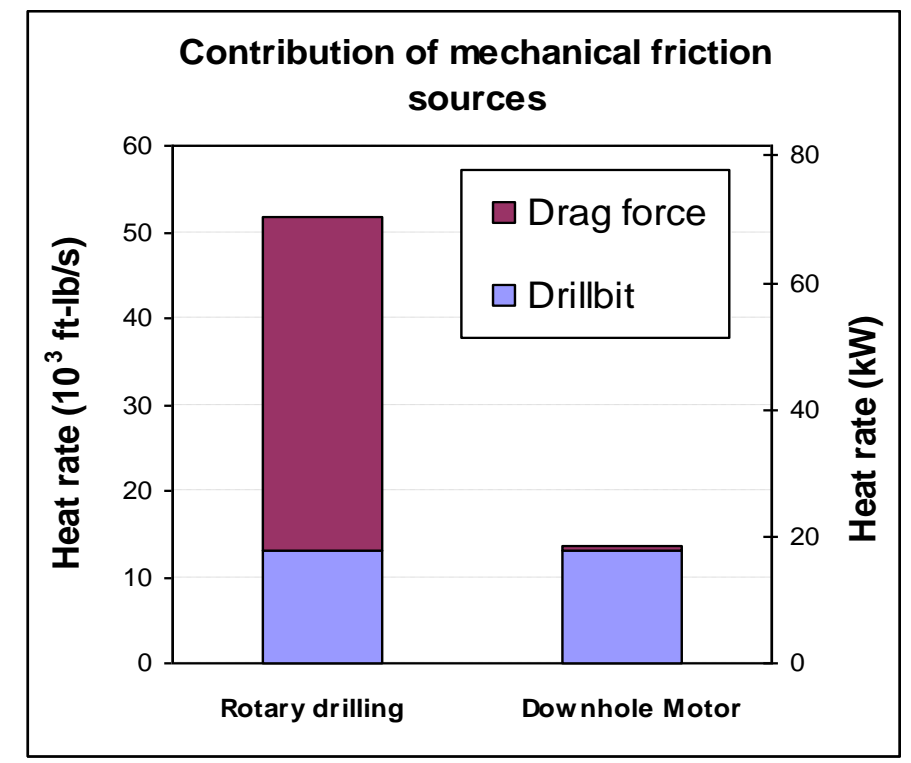

Figure6. Heat generation from mechanical friction

The formation temperature profile is also simulated at different depth using the model. While most of the existing models assume a constant wall temperature, in the model, the transient effect in this model is noticeable in the near wellbore region, especially at different well depths (Figures 7-9). At shallow depth, heating effect (formation temperature increases with time) is observed whereas at higher depth mostly cooling is taking place. These changes in formation temperature alter the thermally induced stresses in the affected region and consequently change the prediction of mud weight window.

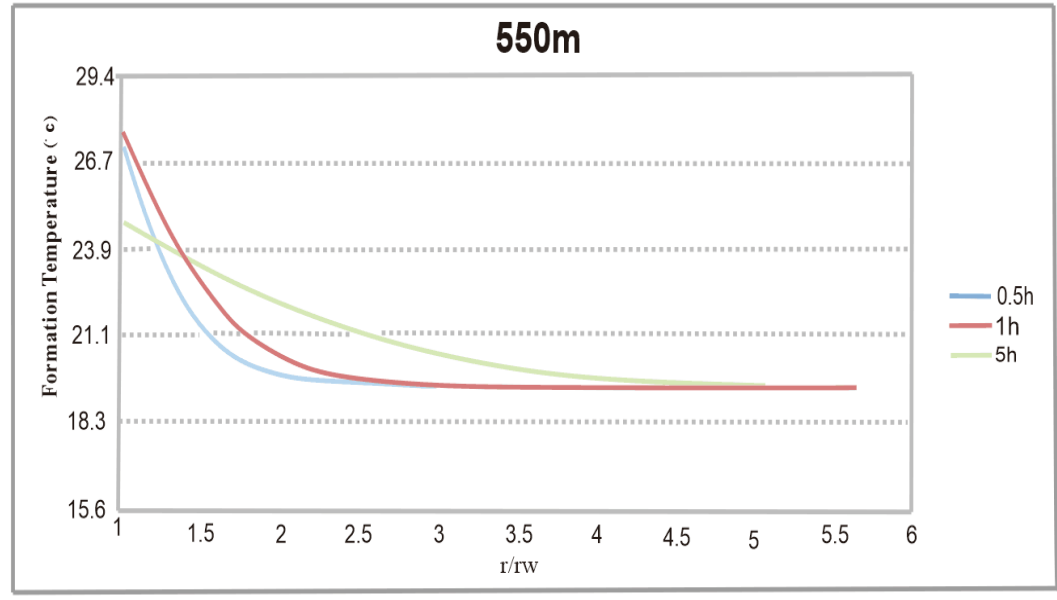

Figure7. Constant Wall Temperatures (Shahabadi-Farahani34 - 2005)

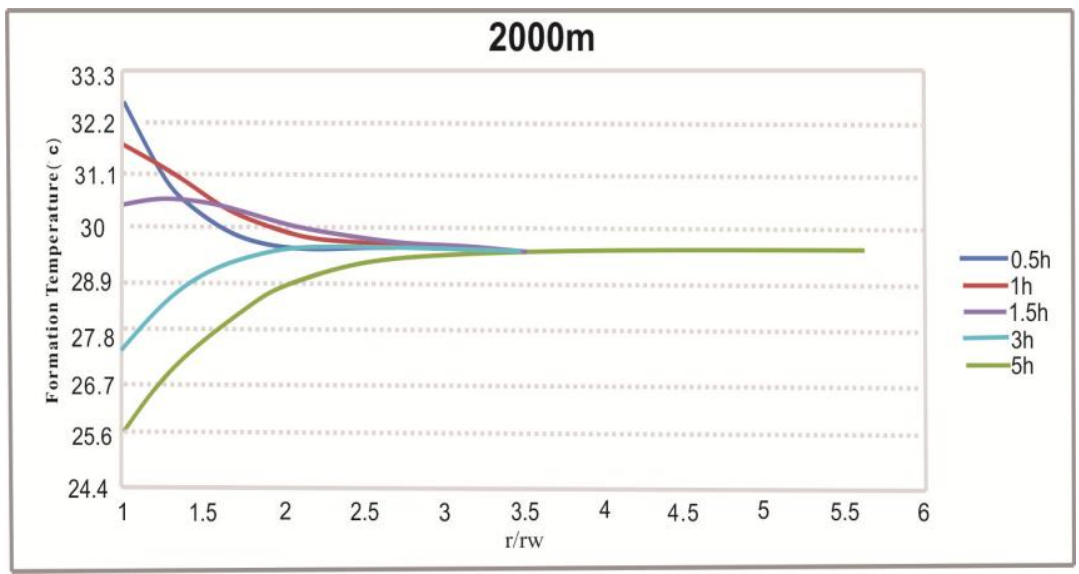

Figure8. Transient Wall Temperature (Medium Depth) 


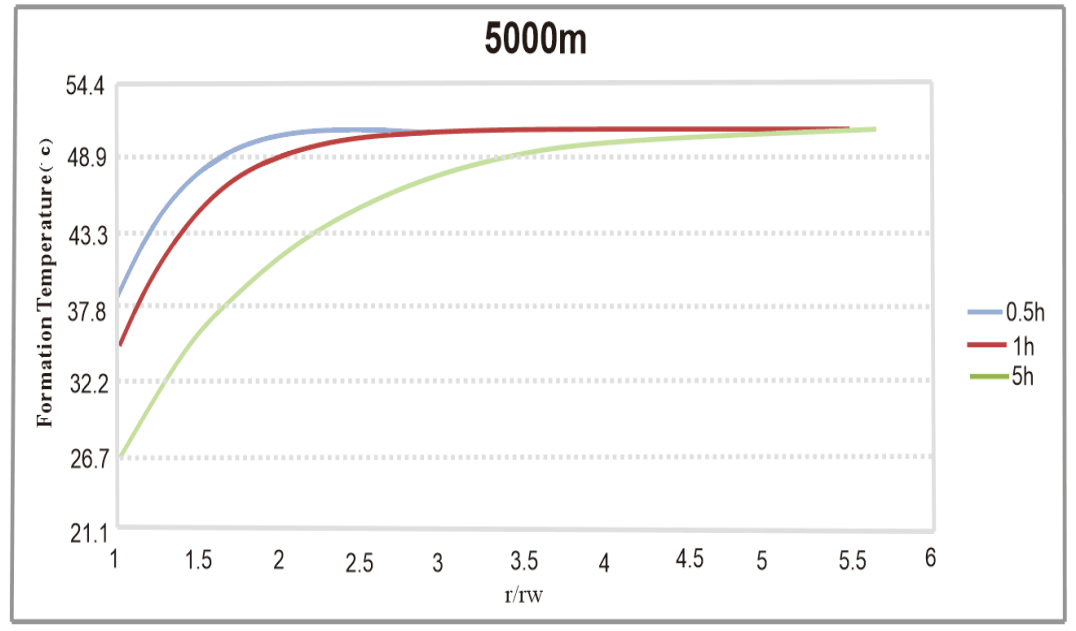

Figure9. Transient Wall Temperature (High Depth)

\section{CONCLUSION}

The following conclusions are reached through the study:

- A new model which considers the effects of the heat created from the contacts between drillpipe and casing/formation is proposed to predict the dynamic wellbore temperature during drilling.

- The new model is utilized in a practical well to study the dynamic temperature changes. Study shows that the majority of additional heat added is due to the drill bit during the sliding mode; the amount of frictional heat generated from drag force is negligible compared to rotary drilling.

- The model can be used to accurate prediction temperature profiles in the wellbore at the design stage or mud and downhole tools selection during drilling operation.

\section{REFERENCES}

[1] Bird, R. Byron, Stewart, Warren E. and Lightfoot, Edwin N.: "Transport Phenomena," New York: John Wiley \& Sons, Inc. (1960).

[2] Chapman, Alan J.: "Heat Transfer," New York: The Macmillan Company (1967).

[3] Chen, Zhongming, and Novotny, Rudolf J.: "Accurate Prediction Wellbore Transient Temperature Profile Under Multiple Temperature Gradients: Finite Difference Approach and Case History,” paper SPE 84583 (2003).

[4] Edwardson, M.J., Girner, H.M., Parkison, H.R., Williams, C.D., and Matthews, C.S.: "Calculation of Formation Temperature Disturbances Caused by Mud Circulation," Journal of Petroleum Technology (April, 1962) 416-426.

[5] Hasan, A.R., and Kabir, C.S.: "Fluid Flow and Heat Transfer in Wellbores," Texas: Society of Petroleum Engineers (2002) 670-674.

[6] Hasan, A.R., Kabir, C.S., Ameen, M.M., and Wang, Xiaowei: "A Mechanistic Model for Circulating Fluid Temperature," Society of Petroleum Engineers Journal (June, 1996) 133-143.

[7] Holman, J.P.: "Heat Transfer," Singapore: McGraw-Hill Book Co. (1989).

[8] Holmes, Charles S., and Swift, Samuel C.: "Calculation of Circulating Mud Temperatures," Journal of Petroleum Technology (June, 1970) 670-674.

[9] Kabir, C.S., Hasan, A.R., Kouba, G.E., and Ameen, M.M.: "Determining Circulating Fluid Temperature in Drilling, Workover, and Well-Control Operations," SPE Drilling \& Completion (June, 1996) 74-79.

[10] Karstad, Eirik: “Analytical Approach to Temperature Induced Well Problems," (Draft - 2006).

[11] Karstad, Eirik, and Aadnøy, Bernt S.: "Analysis of Temperature Measurements during Drilling," paper SPE 38603 (1997).

[12] Keller, H.H., Couch, E.J., and Berry, P.M.: "Temperature Distribution in Circulating Mud Columns," Society of Petroleum Engineers Journal (February, 1973) 23-30.

[13] Maury, Vincent, and Guenot, Alain.: "Practical Advantages of Mud Cooling Systems for Drilling," SPE Drilling \& Completion (March, 1995) 42-48.

[14] Merlo, Antonino, Maglione, Roberto, Piatti, Cesare: "An Innovative Model For Drilling Fluid Hydraulics," paper SPE 29259. 
[15] Mitchell, Robert F., and Sathuvalli, Udaya B.: "Wellbore Thermal and Flow Simulation,” (Draft - 2007).

[16] Ozisik, M. Necati: "Finite Difference Methods in Heat Transfer," Florida: CRC Press, Inc. (1994).

[17] Raymond, L.R.: "Temperature Distribution in a Circulating Drilling Fluid," Journal of Petroleum Technology (March, 1969) 333-341.

[18] Reed, T.D., Pilehvari, A.A.: “A New Model for Laminar, Transitional, and Turbulent Flow of Drilling Muds," paper SPE 25456.

[19] Roy, Ranjit: “A Primer on the Taguchi Method,” New York: Society of Manufacturing (1990).

[20] Skelland, A.H.P.: "Non-Newtonian Flow and Heat Transfer," New York: John Wiley \& Sons, Inc. (1967) 358-433.

[21] Ward, M., Granberry, V., Campos, G., Rausis, M., Sledz, M., Weber, L., Guillot, D., and Romero, J.: “A Joint Industry Project to Assess Circulating Temperatures in Deepwater Wells," paper SPE 71364 (2001).

[22] White, W.W., Zamora, M., and Svoboda, C.F.: "Downhole Measurements of Synthetic-based Drilling Fluid in an Offshore Well Quantify Dynamic Pressure and Temperature Distributions,” paper SPE 35057 (1997).

[23] Wooley, Gary R.: "Computing Downhole Temperatures in Circulation, Injection, and Production Wells," Journal of Petroleum Technology (September, 1980) 1509-1522.

Citation: Ming Jiang, (2019). "A Heat Transfer Model for Accurate Wellbore Temperature Prediction during Drilling”, International Journal of Petroleum and Petrochemical Engineering (IJPPE), 5(4), pp.1-4, DOI: http://dx.doi.org/10.20431/2454-7980.0504001

Copyright: (C) 2019 Authors. This is an open-access article distributed under the terms of the Creative Commons Attribution License, which permits unrestricted use, distribution, and reproduction in any medium, provided the original author and source are credited 